\title{
Chalcones: A Solubility study at different temperatures
}

\author{
Ankita A. Bhalu, Kapil D. Bhesaniya, Nayan J. Vekariya, Shipra H. Baluja* \\ Physical Chemistry Laboratory, Department of Chemistry, Saurashtra University, \\ Rajkot - 360005, Gujarat, India \\ *E-mail address: shipra_baluja@rediffmail.com
}

\begin{abstract}
Some new chalcones are synthesized by the condensation of aryl ketones with aromatic aldehydes and solubility of these synthesized chalcones is determined in chloroform and dichloromethane at temperatures ranging between $293.15 \mathrm{~K}$ and $313.15 \mathrm{~K}$ by gravimetric method. The experimental data was correlated well with modified Apelblat equation. Further, from the experimental solubility data, some thermodynamic parameters such as dissolution enthalpy, Gibb's energy and entropy etc. were evaluated using van't Hoff equation. The positive Gibb's energy and negative entropy suggests spontaneous dissolution and more ordered structure in solution.
\end{abstract}

Keywords: Chloroform, dichloromethane; thermodynamic parameters; dissolution enthalpy; Gibb's energy and entropy

\section{INTRODUCTION}

The chalcones and their derivatives are an interesting class of compounds which are extensively investigated due to their biological and industrial applications. Many of these have been used as important intermediates in organic synthesis [1,2]. The compounds with the backbone of chalcones have been reported to possess various biological activities such as antimicrobial [3-5], antiinflammatory [6,7], antifungal [8,9], antioxidant [10,11], antileishmanial [12], antimalarial [13,14], antituberculosis [15,16], analgesic [17], anti HIV [18], antioxidant [19], antitumor [20,21] and neuro protective [22] activities.

As these compounds are biologically active, it will be interesting to study their solubilities in different solvents. The solubility data has been of fundamental importance in a large number of scientific disciplines and practice applications such as drug discovery, drug formulation, and crystallization based separation etc. Thus, due to biological importance of this class of compounds, in the present study, some new chalcones of vanillin derivatives have been synthesized.

Further, the solubility of these synthesized chalcones was determined in chloroform and dichloromethane at different temperatures (293.15 to $313.15 \mathrm{~K}$ ). From the solubility data, some thermodynamic parameters such as enthalpy, Gibb's energy and entropy of solutions have also been evaluated. 


\section{EXPERIMENTAL}

\section{[A] Synthesis of 3-methoxy-4-ethoxybenzaldehyde:}

An aqueous solution of vanillin $(0.01 \mathrm{M})$ was refluxed at $95{ }^{\circ} \mathrm{C}$ to $97{ }^{\circ} \mathrm{C}$ for half an hour with stirring. To this solution, few drops of sodium hydroxide and diethyl sulphate $(0.012 \mathrm{M})$ were added and reaction mixture was again refluxed for 5 to 7 hours with stirring. The organic layer was isolated and cooled at room temperature. The solid crude product was isolated and crystallized from absolute ethanol.

\section{[B] Synthesis of (2E)-1-(4-ethoxy-3-methoxyphenyl)-3-phenylprop-2-en-1-one:}

A mixture of 3-methoxy-4-ethoxybenzaldehyde and substituted acetophenone in methanol was stirred for 24 hours in presence of few drops of aqueous sodium hydroxide solution. The product was filtered and dried. The recrystalization was done in ethanol. (Scheme I.)

\section{Scheme I}<smiles>COc1cc(C=O)ccc1O</smiles>

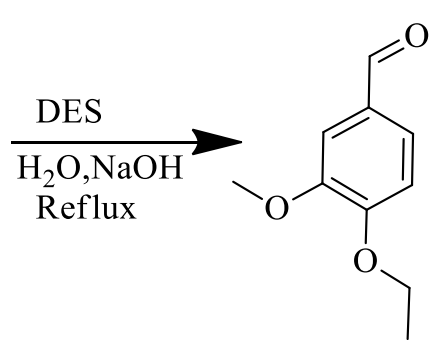

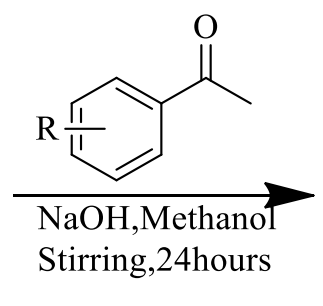

Stirring,24hours<smiles>[R][R]1ccc(/C=C/C(=O)c2ccc(OCC)c(OC)c2)cc1</smiles>

A-1 to A-9

All the synthesized compounds were crystallized and characterization of compounds was done by FTIR, ${ }^{1} \mathrm{H}-\mathrm{NMR}$ and mass spectral data. The melting points of compounds were determined by Differencial Scanning Colorimetry.

\section{SOLUBILITY DETERMINATION}

The solvents; chloroform and dichloromethane were of Allied chemical corporation and were purified by methods reported earlier

The solubility of synthesized compounds was determined in different selected solvents. The gravimetric method was chosen for the investigation. For each measurement, an excess mass of compound was added to a known mass of solvent. The equilibrium cell was heated to a constant temperature with continuous stirring for about $5 \mathrm{~h}$ (the temperature of the water bath approached constant value, and then the actual value of the temperature was recorded). After $5 \mathrm{~h}$, stirring was stopped, and the solution was kept for $2 \mathrm{~h}$ to approach equilibrium.

The equilibrium time of $2 \mathrm{~h}$ is optimized by checking the concentration of solution at different intervals of time. After $2 \mathrm{~h}$, the change in concentration was less than $1 \%$, so saturated solution was assumed to be equilibrium. Upper portion of this clear solution was filtered by a membrane $(0.22 \mu \mathrm{m})$ and kept in a weighed vial. The vial with solution was quickly weighed to determine the mass of the sample and placed in vacuum oven at $323.15 \mathrm{~K}$ to fully evaporate solvents. When the mass of the residue reached at constant value, the final 
mass was recorded. All of the masses were taken using an electronic balance (Mettler Toledo AB204-S, Switzerland) with an uncertainty of \pm 0.0001 g. At each temperature, the measurement was conducted three times, and an average value was used to determine the mole fraction solubility. The saturated mole fraction solubility $(\mathrm{x})$ of compounds in each solvent can be calculated by using equation (1).

$$
x=\frac{\left(m_{2}-m_{0}\right) / M_{1}}{\left(m_{2}-m_{0}\right) / M_{1}+\left(m_{1}-m_{2}\right) / M_{2}}
$$

where $M_{1}$ is the molar mass of chalcones and $M_{2}$ is the molar mass of solvent. At each temperature, the measurement was conducted three times. By using the average value, mole fraction solubility $x$ of chalcones in selected solvents were calculated.

\section{RESULTS AND DISCUSSION}

Table I shows different substitution groups of chalcones [30-39]. The mole fraction solubilities $x$ of chalcones in the chloroform and dichloromethane are listed in Table II at different temperatures ( 293.15 to $313.15 \mathrm{~K}$ ) and more visually given in Figure I and Figure II for chloroform and dichloromethane respectively. In both the solvents, solubility is observed to increase almost linearly with temperature. Further, solubility is greater in dichloro methane than that in chloroform. The temperature dependence solubility has also been described by the modified Apelblat equation [23,24].

$$
\ln x=A+\frac{B}{T}
$$

where, $x$ is the mole fraction solubility of chalcones, $T$ is the absolute temperature. $A$ and $B$ are coefficients. The values of these coefficients are given in Table III. Using these values of $A$ and $B$, the calculated solubilities $\left(x_{c}\right)$ were evaluated and are reported in Table II. Further, relative deviations $(R D)$ between the experimental and calculated values are also evaluated by eq. (3) and are listed in Table II.

$$
R D=\frac{\left(x-x_{c}\right)}{x}
$$

The, relative average deviations $(A R D)$ and root-mean-square deviations (RMSD), calculated by equations (4) and (5) are listed in Tables III.

$$
\begin{aligned}
A R D & =\frac{1}{N} \sum_{i}^{N} \frac{\left(x-x_{c}\right)}{x} \\
R M S D & =\left[\sum_{i=1}^{N} \frac{\left(x_{c}-x\right)^{2}}{N-1}\right]^{\frac{1}{2}}
\end{aligned}
$$

where $N$ is the number of experimental points. 


\section{THERMODYNAMIC FUNCTIONS OF SOLUTION}

The dissolution of a substance in a solvent is associated with changes in thermodynamic functions such as enthalpies of solution $\left(\Delta H_{\text {sol }}\right)$, Gibbs energy change $\left(\Delta G_{\text {sol }}\right)$ and entropy of solutions $\left(\Delta S_{\text {sol }}\right)$. These functions have been evaluated from experimental solubility data. The changes that occur in the solute during dissolution process can be explained by these thermodynamic functions.

In recent thermodynamic treatments, the enthalpies of solution $\left(\Delta H_{s o l}\right)$ was calculated by modified van't Hoff equation [25] i.e., from the slope of the plot of $\ln x$ versus $(1 / T$ $\left.1 / T_{h m}\right)$.

$$
\frac{\partial \ln x}{\partial\left(\frac{1}{T}-\frac{1}{T_{h m}}\right)_{P}}=-\frac{\Delta H_{S o l}}{R}
$$

where $T$ is the experimental temperature, $\Delta H_{\text {sol }}$ is the heat of solution, $R$ is universal gas constant $(8.314 \mathrm{~J} / \mathrm{mol} \mathrm{K}) . T_{h m}$ is mean harmonic temperature which is calculated by the following equation:

$$
T_{h m}=\frac{n}{\sum_{1}^{n \frac{1}{T}}}
$$

where $n$ is the number of experimental temperatures studied. In the present case, the $T_{h m}$ value obtained is $303.15 \mathrm{~K}$. From the intercepts of these plots, Gibbs energy change $\left(\Delta G_{s o l}\right)$ for the solubility process was evaluated by the following relation.

$$
\Delta G_{\text {sol }}=-R \times T_{h m} \times \text { Intercept }
$$

Using these evaluated $\Delta H_{s o l}$ and $\Delta G_{s o l}$ values, the entropies of solutions $\left(\Delta S_{s o l}\right)$ were obtained by the equation .

$$
\Delta S_{s o l}=\frac{\Delta H_{s o l}-\Delta G_{s o l}}{T_{h m}}
$$

\section{NOMENCLATURE}

$M_{1}=$ Molar mass of chalcone.

$M_{2}=$ Molar mass of solvent.

$x=$ Experimental mole fraction solubilities. $\quad x_{c}=$ Calculated mole fraction solubilities.

$A, B$ and $C=$ Coefficients.

$R D=$ Relative deviations.

$A R D=$ Relative average deviations.

$R M S D=$ Root-mean-square deviations.

$N=$ Number of experimental points.

$T=$ Temperature $/ \mathrm{K}$.

$\Delta H_{\text {sol }}=$ Enthalpy change $/ \mathrm{kJ} \cdot \mathrm{mol}^{-1}$.

$\Delta S_{\text {sol }}=$ Entropy change $/ \mathrm{J} \cdot \mathrm{mol}^{-1}$.

$m . f .=$ molecular formula.

$n=$ Number of experimental temperatures studied.

$T_{h m}=$ Mean harmonic temperature.

$\Delta G_{\text {sol }}=$ Gibb's energy change $/ \mathrm{kJ} \cdot \mathrm{mol}^{-1}$.

$R=$ Universal gas constant $/ \mathrm{J} \cdot \mathrm{mol}^{-1} \cdot \mathrm{K}^{-1}$.

$m . p .=$ melting point. 
Table I. Different substitution groups of chalcones.

\begin{tabular}{|c|c|}
\hline $\begin{array}{c}\text { Compound } \\
\text { Code }\end{array}$ & R \\
\hline A-1 & $4-\mathrm{Cl}$ \\
\hline A-2 & $4-\mathrm{NO}_{2}$ \\
\hline A-3 & $-\mathrm{H}$ \\
\hline A-4 & $4-\mathrm{CH}_{3}$ \\
\hline A-5 & $4-\mathrm{OCH}_{3}$ \\
\hline A-6 & $4-\mathrm{Br}$ \\
\hline A-7 & $4-\mathrm{OH}$ \\
\hline A-8 & $2.4-\mathrm{OCH}$ \\
\hline A-9 & $3-\mathrm{Cl}_{3}$ \\
\hline
\end{tabular}

Table II. The experimental solubility $(x)$, calculated solubility $\left(x_{c}\right)$ and relative deviation $(R D)$ of chalcones in chloroform and dichloromethane (DCM) at different temperatures.

\begin{tabular}{|c|c|c|c|c|c|c|}
\hline \multirow{2}{*}{$T / K$} & \multicolumn{3}{|c|}{ Chloroform } & \multicolumn{3}{|c|}{ DCM } \\
\hline & $x .10^{3}$ & $x_{c} 10^{3}$ & 100RD & $x .10^{3}$ & $x_{c \cdot} 10^{3}$ & 100RD \\
\hline & \multicolumn{3}{|c|}{ A-1 } & \multicolumn{3}{|c|}{ A-1 } \\
\hline 293.15 & 5.6660 & 5.1774 & 8.6230 & 8.9330 & 10.0937 & -12.9851 \\
\hline 298.15 & 6.5141 & 5.8374 & 10.3886 & 14.6171 & 13.2873 & 9.1010 \\
\hline 303.15 & 7.3912 & 6.5815 & 10.9554 & 19.5442 & 17.4921 & 10.5013 \\
\hline 305.15 & 8.2940 & 7.4204 & 10.5327 & 23.8635 & 23.0272 & 3.5010 \\
\hline \multirow[t]{2}{*}{313.15} & 9.2345 & 8.3663 & 9.4014 & 27.6761 & 30.3153 & -9.5339 \\
\hline & \multicolumn{3}{|c|}{ A-2 } & \multicolumn{3}{|c|}{ A-2 } \\
\hline 293.15 & 0.8125 & 0.7866 & 3.1948 & 3.5648 & 3.4788 & 2.4138 \\
\hline 298.15 & 0.8699 & 0.8394 & 3.5077 & 6.1866 & 4.8386 & 21.7894 \\
\hline 303.15 & 0.9286 & 0.8958 & 3.5378 & 8.7791 & 6.7299 & 23.3417 \\
\hline 305.15 & 0.9936 & 0.9559 & 3.7940 & 11.350 & 9.3605 & 17.5256 \\
\hline 313.15 & 1.0552 & 1.0201 & 3.3239 & 13.8922 & 13.019 & 6.2788 \\
\hline
\end{tabular}




\begin{tabular}{|c|c|c|c|c|c|c|}
\hline & \multicolumn{3}{|c|}{ A-3 } & \multicolumn{3}{|c|}{ A-3 } \\
\hline 293.15 & 3.7360 & 3.1901 & 14.6139 & 1.0250 & 1.2626 & -23.1777 \\
\hline 298.15 & 4.0605 & 3.4730 & 14.4687 & 2.3081 & 1.9398 & 15.9599 \\
\hline 303.15 & 4.3825 & 3.7811 & 13.7226 & 3.5770 & 2.9802 & 16.6842 \\
\hline 305.15 & 4.7020 & 4.1165 & 12.4571 & 4.8318 & 4.5787 & 5.2377 \\
\hline \multirow[t]{2}{*}{313.15} & 5.0191 & 4.4816 & 10.7085 & 6.0726 & 7.0345 & -15.8408 \\
\hline & \multicolumn{3}{|c|}{ A-4 } & \multicolumn{3}{|c|}{ A-4 } \\
\hline 293.15 & 0.3365 & 0.5229 & -55.4173 & 20.7421 & 23.3183 & -12.4199 \\
\hline 298.15 & 1.6490 & 1.0066 & 38.9531 & 26.7072 & 27.3631 & 2.4564 \\
\hline 303.15 & 2.9570 & 1.9377 & 34.4687 & 31.8882 & 32.1105 & -0.6956 \\
\hline 305.15 & 4.2650 & 3.7300 & 12.5439 & 38.8004 & 37.6811 & 2.8859 \\
\hline \multirow[t]{2}{*}{313.15} & 5.5685 & 7.1799 & -28.9394 & 44.9301 & 44.2171 & 1.5863 \\
\hline & \multicolumn{3}{|c|}{ A-5 } & \multicolumn{3}{|c|}{ A-5 } \\
\hline 293.15 & 6.2033 & 6.7354 & -8.5778 & 0.7198 & 0.8754 & -21.4893 \\
\hline 298.15 & 6.6599 & 7.1340 & -7.1192 & 1.4667 & 1.2860 & 12.283 \\
\hline 303.15 & 7.1025 & 7.5415 & -6.3874 & 2.1875 & 1.8924 & 13.4899 \\
\hline 305.15 & 7.5415 & 8.0033 & -6.1236 & 2.8768 & 2.8768 & 3.2358 \\
\hline \multirow[t]{2}{*}{313.15} & 7.9670 & 8.4769 & -6.3993 & 3.5398 & 4.0948 & -15.6789 \\
\hline & \multicolumn{3}{|c|}{ A-6 } & \multicolumn{3}{|c|}{ A-6 } \\
\hline 293.15 & 6.0741 & 6.6551 & -9.5651 & 3.3644 & 3.916 & -16.4136 \\
\hline 298.15 & 9.7428 & 9.1095 & 6.5002 & 5.9615 & 5.9615 & 7.7959 \\
\hline 303.15 & 13.5748 & 13.5748 & 8.1441 & 8.5123 & 7.7145 & 9.3724 \\
\hline 305.15 & 17.5713 & 17.0681 & 2.8640 & 11.012 & 10.827 & 1.6766 \\
\hline \multirow[t]{2}{*}{313.15} & 21.7481 & 23.3630 & -7.4255 & 13.467 & 15.195 & -12.8318 \\
\hline & \multicolumn{3}{|c|}{ A-7 } & \multicolumn{3}{|c|}{ A-7 } \\
\hline 293.15 & 6.5624 & 5.1287 & 21.8470 & 2.0630 & 1.7280 & 16.2401 \\
\hline 298.15 & 7.4556 & 5.6964 & 23.5957 & 2.9055 & 2.1856 & 24.7747 \\
\hline 303.15 & 8.3636 & 6.3269 & 24.3510 & 3.7936 & 2.7645 & 27.1277 \\
\hline
\end{tabular}




\begin{tabular}{|l|ccc|ccc|}
\hline 305.15 & 9.2687 & 7.0273 & 24.1828 & 4.1240 & 3.4967 & 15.2110 \\
\hline 313.15 & 10.180 & 7.8051 & 23.3281 & 4.4228 & 4.4228 & 22.7194 \\
\hline & \multicolumn{3}{|c|}{$\mathbf{A - 8}$} & & & $\mathbf{A - 8}$ \\
\hline 293.15 & 9.9591 & 8.6000 & 13.6468 & 4.5115 & 3.6677 & 18.7033 \\
\hline 298.15 & 10.0359 & 8.6431 & 13.8781 & 5.4687 & 4.3040 & 21.2977 \\
\hline 303.15 & 10.1120 & 8.6864 & 14.0980 & 6.4837 & 5.0506 & 22.1028 \\
\hline 305.15 & 10.1880 & 8.7299 & 14.3110 & 7.5618 & 5.9268 & 21.6221 \\
\hline 313.15 & 10.2632 & 8.7737 & 14.5131 & 8.7093 & 6.9549 & 20.1434 \\
\hline & & $\mathbf{A - 9}$ & & & $\mathbf{A - 9}$ & \\
\hline 293.15 & 16.321 & 15.020 & 15.020 & 9.2359 & 8.5974 & 6.9128 \\
\hline 298.15 & 15.899 & 15.478 & 15.478 & 11.891 & 10.448 & 12.1311 \\
\hline 303.15 & 16.417 & 15.949 & 15.949 & 14.639 & 12.697 & 13.2639 \\
\hline 305.15 & 16.924 & 16.435 & 16.435 & 17.500 & 15.431 & 11.8256 \\
\hline 313.15 & 18.458 & 16.2489 & 16.935 & 20.380 & 18.752 & 7.9841 \\
\hline
\end{tabular}

Table III. Coefficients A and B of equation (1), Relative Average Deviation (ARD), Root Mean Square Deviation $(R M S D)$ of Chalcones in Chloroform and Dichloromethane.

\begin{tabular}{|c|c|c|c|c|}
\hline $\begin{array}{c}\text { Compound } \\
\text { code }\end{array}$ & A & B & $\mathbf{1 0}^{\mathbf{7}}$ RMDS & 100ARD \\
\hline \multicolumn{5}{|c|}{ Chloroform } \\
\hline A-1 & -12.300 & 0.024 & 1.4345 & 9.9798 \\
\hline A-2 & -10.960 & 0.013 & 0.0026 & 3.4716 \\
\hline A-3 & -10.732 & 0.017 & 0.8183 & 13.1933 \\
\hline A-4 & -45.960 & 0.131 & 2.1847 & 0.3218 \\
\hline A-5 & -8.373 & 0.012 & 0.5934 & -6.9214 \\
\hline A-6 & -23.423 & 0.063 & 2.4110 & 0.1034 \\
\hline A-7 & 11.430 & 0.021 & 9.9808 & 23.4609 \\
\hline A-8 & -5.050 & -5.958 & 0.001 & 5.0819 \\
\hline A-9 & & 0.006 & 2.3224 & 4.9211 \\
\hline & -20.72 & 0.055 & 7.4939 \\
\hline A-1 & & & \\
\hline
\end{tabular}




\begin{tabular}{|l|llll|}
\hline A-2 & -25.01 & 0.066 & 5.3705 & 14.2698 \\
\hline A-3 & -31.85 & 0.085 & 0.7688 & -0.2273 \\
\hline A-4 & -13.14 & 0.032 & 4.4389 & -2.2199 \\
\hline A-5 & 29.67 & 0.077 & 0.2300 & -1.6318 \\
\hline A-6 & -25.41 & 0.067 & 2.0889 & -2.0800 \\
\hline A-7 & -20.14 & 0.047 & 1.8868 & 21.2145 \\
\hline A-8 & -14.99 & 0.032 & 4.9366 & 20.7738 \\
\hline A-9 & -16.19 & 0.039 & 6.5944 & 10.4234 \\
\hline
\end{tabular}

Table IV. The thermodynamic parameters of chalcones in chloroform and dichloromethane (DCM).

\begin{tabular}{|c|c|c|c|c|c|c|}
\hline $\begin{array}{c}\text { Compound } \\
\text { Code }\end{array}$ & $\begin{array}{c}\Delta \mathbf{H}_{\text {sol }} \\
/ \mathbf{k J} \cdot \mathbf{m o l}^{-1} \\
\end{array}$ & $\Delta \mathbf{G}_{\mathrm{sol}} / \mathbf{k J} \cdot \mathrm{mol}^{-1}$ & $\Delta \mathbf{S}_{\mathrm{sol}} / \mathbf{J} \cdot \mathbf{m o l}^{-1}$ & $\begin{array}{c}\Delta \mathbf{H}_{\text {sol }} \\
/ \mathbf{k J} \cdot \mathbf{m o l}^{-1} \\
\end{array}$ & $\begin{array}{c}\Delta \mathbf{G}_{\text {sol }} \\
/ \mathbf{k J} \cdot \mathbf{m o l}^{-1}\end{array}$ & $\Delta \mathbf{S}_{\mathrm{sol}} / \mathbf{J} \cdot \mathbf{m o l}^{-1}$ \\
\hline & \multicolumn{3}{|c|}{ Chloroform } & \multicolumn{3}{|c|}{ DCM } \\
\hline A-1 & 0.0019 & 12.3839 & -40.8846 & 0.0042 & 10.1776 & -33.5771 \\
\hline A-2 & 19.1887 & 19.5098 & -1.0597 & 50.9731 & 12.1971 & 127.9801 \\
\hline A-3 & 12.9948 & 14.0360 & -3.4364 & 65.9051 & 14.6204 & 169.2648 \\
\hline A-4 & 100.8821 & 15.5675 & 281.5801 & 24.9370 & 8.7914 & 53.2886 \\
\hline A-5 & 9.5362 & 12.4742 & -9.6969 & 59.1791 & 12.2903 & 154.7567 \\
\hline A-6 & 48.079 & 11.0333 & 122.2719 & 51.9043 & 12.2903 & 130.7458 \\
\hline A-7 & 16.7361 & 12.0787 & 15.3717 & 36.5400 & 14.2324 & 73.6260 \\
\hline A-8 & 1.1473 & 11.5724 & -34.4078 & 25.0501 & 12.7336 & 40.6504 \\
\hline A-9 & 5.1522 & 10.2801 & -16.9247 & 29.8888 & 10.7260 & 63.2469 \\
\hline
\end{tabular}

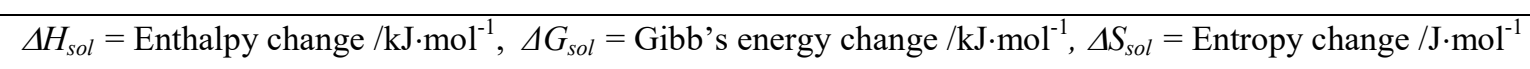




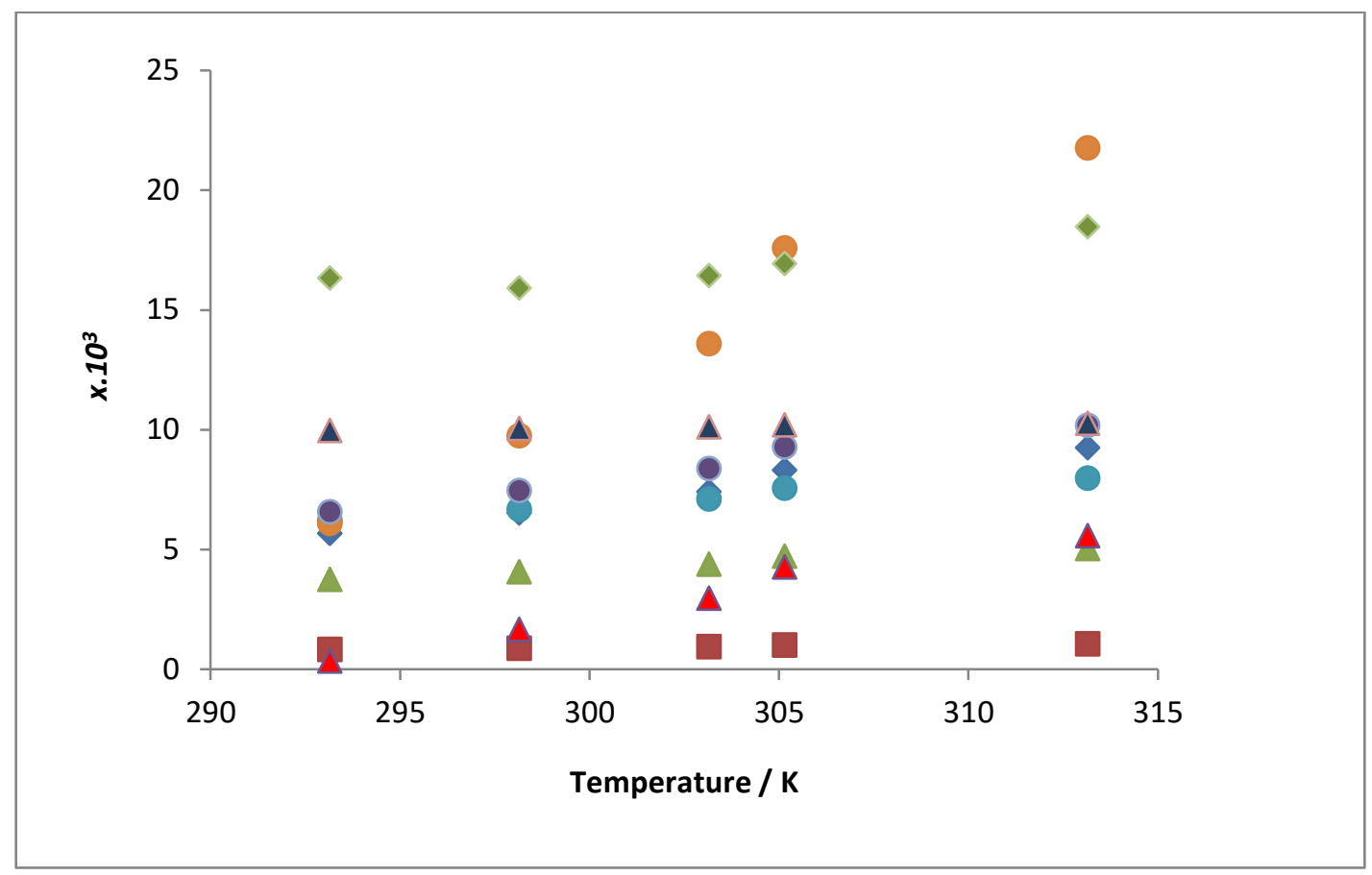

Figure I. The mole fraction solubility $(x)$ against temperature for Chalcones in Chloroform. $\bullet \mathrm{A}-1 ; \sim \mathrm{A}-2 ; \Delta \mathrm{A}-3 ; \Delta \mathrm{A}-4 ; \bullet \mathrm{A}-5 ; \bullet \mathrm{A}-6 ; \bullet \mathrm{A}-7 ; \Delta \mathrm{A}-8 ; \bullet \mathrm{A}-9$.

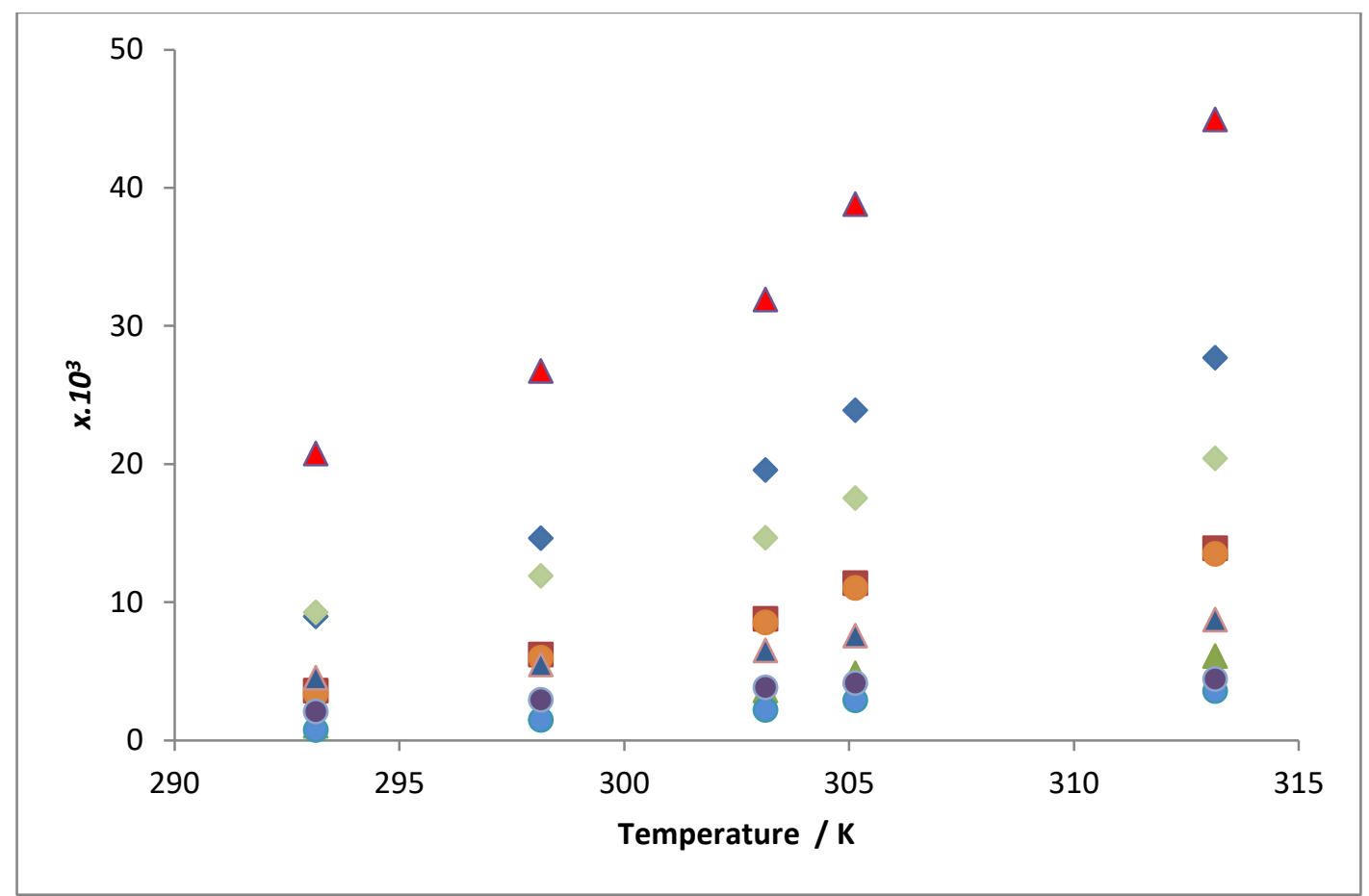

Figure II. The mole fraction solubility $(\mathrm{x})$ against temperature for chalcones in dichloromethane. $\bullet \mathrm{A}-1 ; \bullet \mathrm{A}-2 ; \Delta \mathrm{A}-3 ; \Delta \mathrm{A}-4 ; \bullet \mathrm{A}-5 ; \bullet \mathrm{A}-6 ; \bullet \mathrm{A}-7 ; \Delta \mathrm{A}-8 ; \bullet \mathrm{A}-9$. 


\section{ANALYTICAL AND SPECTRAL DATA}

A-1: Yield: $73 \%$; m.p. $153{ }^{\circ} \mathrm{C}$; m.f. $\mathrm{C}_{18} \mathrm{H}_{17} \mathrm{ClO}_{3}$; IR $\left(\mathrm{KBr}, \mathrm{cm}^{1}\right)$ : $1512.32(\mathrm{C}=\mathrm{C}$ stretching), $1653.73(\mathrm{C}=\mathrm{O}$ stretching), 1232.54 (C-O-C stretching), 2958 (C-H stretching), 844 (C-Cl stretching), ${ }^{1} \mathrm{H}$ NMR (BRUCKER Spectrometer $400 \mathrm{MHz}$ DMSO- $\left.d_{6}, \delta \mathrm{ppm}\right): 3.34(3 \mathrm{H}, \mathrm{s},-$ $\left.\mathrm{OCH}_{3}\right), 4.01-4.08\left(2 \mathrm{H}, \mathrm{q},-\mathrm{OCH}_{2}\right), 1.30-1.35\left(3 \mathrm{H}, \mathrm{t},-\mathrm{CH}_{3}\right), 6.96-6.99(1 \mathrm{H}, \mathrm{d}$, aromatic $), 7.34-$ $7.37(1 \mathrm{H}, \mathrm{d}$, aromatic), $7.53(1 \mathrm{H}, \mathrm{s}$, aromatic), 8.14-8.17 $(2 \mathrm{H}, \mathrm{s},-\mathrm{CH}-\mathrm{CH}), 7.60-7.63(2 \mathrm{H}, \mathrm{d}$, aromatic), 7.72-7.83 (2H, d, aromatic); MS: (m/z): $316\left(\mathrm{M}^{+} \mathrm{BP}, 100 \%\right), 288,282,254,180$, 155.

A-2: Yield: 59 \%; m.p. $153{ }^{\circ} \mathrm{C}$; m.f. $\mathrm{C}_{18} \mathrm{H}_{17} \mathrm{NO}_{5}$; IR $\left(\mathrm{KBr}, \mathrm{cm}^{1}\right)$ : $1495(\mathrm{C}=\mathrm{C}$ stretching), 1678 (C=O stretching), 1248 (C-O-C stretching), 2953 (C-H stretching), 1318 (C-N stretching); ${ }^{1} \mathrm{H}$ NMR (BRUCKER Spectrometer $400 \mathrm{MHz}$ DMSO- $d_{6}, \delta$ ppm): $3.28\left(3 \mathrm{H}, \mathrm{s},-\mathrm{OCH}_{3}\right), 3.98-$ $4.05\left(2 \mathrm{H}, \mathrm{q},-\mathrm{OCH}_{2}\right), 1.32-1.37\left(3 \mathrm{H}, \mathrm{t},-\mathrm{CH}_{3}\right), 6.92-7.03(1 \mathrm{H}, \mathrm{d}$, aromatic $), 7.32(1 \mathrm{H}, \mathrm{d}$, aromatic), $7.33(1 \mathrm{H}, \mathrm{s}$, aromatic), $7.86-8.18(2 \mathrm{H}, \mathrm{s},-\mathrm{CH}-\mathrm{CH}), 7.93(2 \mathrm{H}, \mathrm{d}$, aromatic), 8.02 (2H, d, aromatic); MS: (m/z): $327\left(\mathrm{M}^{+} \mathrm{BP}, 100 \%\right), 298,281,176,151$.

A-3: Yield: $66 \%$; m.p. $132{ }^{\circ} \mathrm{C}$; m.f $\mathrm{C}_{18} \mathrm{H}_{18} \mathrm{O}_{3}$; $\mathrm{IR}\left(\mathrm{KBr}, \mathrm{cm}^{1}\right)$ : $1488.64(\mathrm{C}=\mathrm{C}$ stretching), 1682.32 ( $\mathrm{C}=\mathrm{O}$ stretching), 1257 (C-O-C stretching), 2955 (C-H asymmetrical stretching), ${ }^{1} \mathrm{H}$ NMR (BRUCKER Spectrometer $400 \mathrm{MHz}$ DMSO- $\left.d_{6}, \delta \mathrm{ppm}\right): 3.23\left(3 \mathrm{H}, \mathrm{s},-\mathrm{OCH}_{3}\right), 4.05-$ $4.10\left(2 \mathrm{H}, \mathrm{q},-\mathrm{OCH}_{2}\right), 1.23-1.28\left(3 \mathrm{H}, \mathrm{t},-\mathrm{CH}_{3}\right), 7.01-7.03(1 \mathrm{H}, \mathrm{d}$, aromatic $), 7.40-7.43(1 \mathrm{H}, \mathrm{d}$, aromatic), $7.58(1 \mathrm{H}, \mathrm{s}$, aromatic), 8.24-8.27 (2H, s, $-\mathrm{CH}-\mathrm{CH}), 7.60-7.63(2 \mathrm{H}, \mathrm{d}$, aromatic), 7.65-7.73 $\left(2 \mathrm{H}, \mathrm{d}\right.$, aromatic), $7.59\left(1 \mathrm{H}, \mathrm{s}\right.$, aromatic); MS: $(\mathrm{m} / \mathrm{z}): 282\left(\mathrm{M}^{+} \mathrm{BP}, 100 \%\right), 282$, $267,253,237,151,131$.

A-4: Yield: $67 \%$ m.p. $124{ }^{\circ} \mathrm{C}$; m.f. $\mathrm{C}_{19} \mathrm{H}_{20} \mathrm{O}_{4}$; IR $\left(\mathrm{KBr}, \mathrm{cm}^{-1}\right): 1522.31(\mathrm{C}=\mathrm{C}$ stretching), 1642.47 ( $\mathrm{C}=\mathrm{O}$ stretching), 1269.05 (C-O-C stretching), 2922 (C-H stretching), 2953 (C-C retching), ${ }^{1} \mathrm{H}$ NMR (BRUCKER Spectrometer $400 \mathrm{MHz}$ DMSO-d, $\left.\delta \mathrm{ppm}\right): 3.29$ (3H, s, $\left.\mathrm{OCH}_{3}\right), 4.03-4.10\left(2 \mathrm{H}, \mathrm{q},-\mathrm{OCH}_{2}\right), 1.21-1.32\left(3 \mathrm{H}, \mathrm{t}, \mathrm{CH}_{3}\right), 6.90-6.96(1 \mathrm{H}, \mathrm{d}$, aromatic $), 728-$ $7.32(1 \mathrm{H}, \mathrm{d}$, aromatic), $7.49(1 \mathrm{H}, \mathrm{s}$, aromatic $), 8.12-8.15(2 \mathrm{H}, \mathrm{s},-\mathrm{CH}-\mathrm{CH}), 7.58-7.61(2 \mathrm{H}, \mathrm{d}$, aromatic), 7.55-7.62 (2H, d, aromatic); $2.52\left(3 \mathrm{H}, \mathrm{d},-\mathrm{CH}_{3}\right) ; \mathrm{MS}:(\mathrm{m} / \mathrm{z}): 296\left(\mathrm{M}^{+} \mathrm{BP}, 100 \%\right)$, $281,267,251,151$.

A-5: Yield: $73 \%$; m.p. $151^{\circ} \mathrm{C}$; m.f. $\mathrm{C}_{18} \mathrm{H}_{20} \mathrm{O}_{4}$; $\mathrm{IR}\left(\mathrm{KBr}, \mathrm{cm}^{1}\right)$ : $1512.32(\mathrm{C}=\mathrm{C}$ stretching), 1665.14 (C=O stretching), 1273.05 (C-O-C stretching), 2977 (C-H stretching), 1276 (C-O-C stretching); ${ }^{1} \mathrm{H}$ NMR (BRUCKER Spectrometer $400 \mathrm{MHz}$ DMSO- $\left.d_{6}, \delta \mathrm{ppm}\right): 3.26(3 \mathrm{H}, \mathrm{s},-$ $\left.\mathrm{OCH}_{3}\right), 3.96-4.03\left(2 \mathrm{H}, \mathrm{q},-\mathrm{OCH}_{2}\right), 1.33-1.38\left(3 \mathrm{H}, \mathrm{t}, \mathrm{CH}_{3}\right), 6.90-6.93(1 \mathrm{H}, \mathrm{d}$, aromatic), 7.30$7.34(1 \mathrm{H}, \mathrm{d}$, aromatic), $7.58(1 \mathrm{H}, \mathrm{s}$, aromatic $), 8.13-8.16(2 \mathrm{H}, \mathrm{s},-\mathrm{CH}-\mathrm{CH}), 7.63-7.66(2 \mathrm{H}, \mathrm{d}$, aromatic), $7.90-7.93\left(2 \mathrm{H}, \mathrm{d}\right.$, aromatic), $3.93\left(3 \mathrm{H}, \mathrm{s},-\mathrm{OCH}_{3}\right)$; $\mathrm{MS}:(\mathrm{m} / \mathrm{z}): 312\left(\mathrm{M}^{+} \mathrm{BP}, 100 \%\right)$, 283, 281, 197, 168, 151.

A-6: Yield: $57 \%$; m.p. $134{ }^{\circ} \mathrm{C}$; m.f $\mathrm{C}_{18} \mathrm{H}_{17} \mathrm{BrO}_{3}$; $\mathrm{IR}\left(\mathrm{KBr}, \mathrm{cm}^{1}\right)$ : $1524.85(\mathrm{C}=\mathrm{C}$ stretching), 1654.84 ( $\mathrm{C}=\mathrm{O}$ stretching), 1248.21 (C-O-C stretching), 2967 (C-H stretching), 778 (C-Br stretching); ${ }^{1} \mathrm{H}$ NMR (BRUCKER Spectrometer $400 \mathrm{MHz}$ DMSO- $d_{6}, \delta$ ppm): $3.23(3 \mathrm{H}, \mathrm{s},-$ $\left.\mathrm{OCH}_{3}\right), 4.05-4.10\left(2 \mathrm{H}, \mathrm{q}, \mathrm{OCH}_{2}\right), 1.23-1.28\left(3 \mathrm{H}, \mathrm{t}, \mathrm{CH}_{3}\right), 7.01-7.03(1 \mathrm{H}, \mathrm{d}$, aromatic), 7.40$7.43(1 \mathrm{H}, \mathrm{d}$, aromatic), $7.58(1 \mathrm{H}, \mathrm{s}$, aromatic), 8.24-8.27 $(2 \mathrm{H}, \mathrm{s},-\mathrm{CH}-\mathrm{CH}), 7.60-7.63(2 \mathrm{H}, \mathrm{d}$, aromatic), 7.65-7.73 (2H ,d, aromatic), $7.59\left(1 \mathrm{H}, \mathrm{s}\right.$, aromatic); $\mathrm{MS}:(\mathrm{m} / \mathrm{z}): 361\left(\mathrm{M}^{+} \mathrm{BP}, 100\right.$ $\%), 345,331,329,208,151$. 
A-7: Yield: $59 \%$; m.p. $133{ }^{\circ} \mathrm{C}$; m.f. $\mathrm{C}_{18} \mathrm{H}_{18} \mathrm{O}_{4}$; IR $\left(\mathrm{KBr}, \mathrm{cm}^{1}\right)$ : $1502.32(\mathrm{C}=\mathrm{C}$ stretching), 1662.14 ( $\mathrm{C}=\mathrm{O}$ stretching), 1270.05 (C-O-C stretching), 2973 (C-H stretching), 3412(O-H stretching); ${ }^{1} \mathrm{H}$ NMR (BRUCKER Spectrometer $400 \mathrm{MHz}$ DMSO- $\left.d_{6}, \delta \mathrm{ppm}\right): 3.44(3 \mathrm{H}, \mathrm{s},-$ $\left.\mathrm{OCH}_{3}\right), 4.03-4.12\left(2 \mathrm{H}, \mathrm{q},-\mathrm{OCH}_{2}\right), 1.38-1.42\left(3 \mathrm{H}, \mathrm{t}, \mathrm{CH}_{3}\right), 7.02-7.10(1 \mathrm{H}, \mathrm{d}$, aromatic $), 7.25-$ $7.28(1 \mathrm{H}, \mathrm{d}$, aromatic), $7.50(1 \mathrm{H}, \mathrm{s}$, aromatic), 8.16-8.20 $(2 \mathrm{H}, \mathrm{s},-\mathrm{CH}-\mathrm{CH}), 7.58-7.68(2 \mathrm{H}, \mathrm{d}$, aromatic), 7.75-7.86 $\left(2 \mathrm{H}, \mathrm{d}\right.$, aromatic), $5.40(1 \mathrm{H}, \mathrm{s},-\mathrm{OH}) ; \mathrm{MS}:(\mathrm{m} / \mathrm{z}): 281\left(\mathrm{M}^{+} \mathrm{BP}, 100 \%\right)$, 269, 205, 151, 157.

A-8: Yield: $64 \%$; m.p. $129{ }^{\circ} \mathrm{C}$; m.f. $\mathrm{C}_{20} \mathrm{H}_{22} \mathrm{O}_{5}$; IR $\left(\mathrm{KBr}, \mathrm{cm}^{1}\right)$ : $1518.12(\mathrm{C}=\mathrm{C}$ stretching), 1677.65 (C=O stretching), 1272.42 (C-O-C stretching), 2912 (C-H stretching), 1231 (C-O-C stretching); ${ }^{1} \mathrm{H}$ NMR (BRUCKER Spectrometer $400 \mathrm{MHz}$ DMSO- $\left.d_{6}, \delta \mathrm{ppm}\right): 3.32(3 \mathrm{H}, \mathrm{s},-$ $\left.\mathrm{OCH}_{3}\right), 4.15-4.21\left(2 \mathrm{H}, \mathrm{q},-\mathrm{OCH}_{2}\right), 1.28-1.32\left(3 \mathrm{H}, \mathrm{t}, \mathrm{CH}_{3}\right), 7.03-7.12(1 \mathrm{H}, \mathrm{d}$, aromatic), 7.34$7.37(1 \mathrm{H}, \mathrm{d}$,aromatic), $7.53(1 \mathrm{H}, \mathrm{s}$, aromatic), 8.20-8.24 $(2 \mathrm{H}, \mathrm{s},-\mathrm{CH}-\mathrm{CH}), 7.58-7.62(1 \mathrm{H}, \mathrm{d}$, aromatic), 7.72-7.83 $\left(2 \mathrm{H}, \mathrm{d}\right.$, aromatic), $7.70-7.75(1 \mathrm{H}, \mathrm{s}$, aromatic $), 3.88\left(3 \mathrm{H}, \mathrm{s},-\mathrm{OCH}_{3}\right), 3.83$ $\left(3 \mathrm{H}, \mathrm{s},-\mathrm{OCH}_{3}\right)$; MS: (m/z): $327\left(\mathrm{M}^{+} \mathrm{BP}, 100 \%\right), 297,280,311,151$.

A-9: Yield: $75 \%$; m.p. $170{ }^{\circ} \mathrm{C}$; m.f. $\mathrm{C}_{18} \mathrm{H}_{17} \mathrm{ClO}_{3}$; IR $\left(\mathrm{KBr}, \mathrm{cm}^{1}\right)$ : $1498.64(\mathrm{C}=\mathrm{C}$ stretching), 1679.12 ( $\mathrm{C}=\mathrm{O}$ stretching), 1243.65 (C-O-C stretching), 2970 (C-H stretching), 776(C-Cl stretching); ${ }^{1} \mathrm{H}$ NMR (BRUCKER Spectrometer $400 \mathrm{MHz}$ DMSO- $d_{6}, \delta$ ppm): $3.43(3 \mathrm{H}, \mathrm{s},-$ $\left.\mathrm{OCH}_{3}\right), 4.13-4.19\left(2 \mathrm{H}, \mathrm{q},-\mathrm{OCH}_{2}\right), 1.26-1.31\left(3 \mathrm{H}, \mathrm{t},-\mathrm{CH}_{3}\right), 6.84-6.92(1 \mathrm{H}, \mathrm{d}$, aromatic), 730$7.35(1 \mathrm{H}, \mathrm{d}$, aromatic), $7.47(1 \mathrm{H}, \mathrm{s}$, aromatic $), 8.12-8.16(2 \mathrm{H}, \mathrm{s},-\mathrm{CH}-\mathrm{CH}), 7.60-7.63(1 \mathrm{H}, \mathrm{d}$, aromatic), 7.72-7.83 $(2 \mathrm{H}, \mathrm{d}$, aromatic), $7.79-7.83(1 \mathrm{H}, \mathrm{t}$, aromatic), $7.65(1 \mathrm{H}, \mathrm{d}$, aromatic), $7.52\left(1 \mathrm{H}, \mathrm{s}\right.$, aromatic); MS: (m/z): $327\left(\mathrm{M}^{+} \mathrm{BP}, 100 \%\right), 288,282,254,180,155$.

\section{CONCLUSIONS}

It is evident from thermodynamic functions are reported in Table IV that for all the compounds, $\Delta H_{\text {sol }}$ and $\Delta G_{\text {sol }}$ values are positive where as $\Delta S_{\text {sol }}$ values are both positive and negative for both the solvents. When stronger bonds are broken and weaker bonds are formed, energy is consumed and so $\Delta H_{\text {sol }}$ becomes positive [26].

This indicates endothermic dissolutions of compounds. The positive values of $\Delta \mathrm{G}_{\mathrm{sol}}$ indicate that the dissolution process is spontaneous. The negative entropy suggests more ordered structure in solution [29] whereas positive entropy is due to more randomness in solution.

As reported in Table I, all the compounds have same central moiety but different side chains, due to different in side chains behave differently in different solutions upon dissolution. Thus, different types of interactions affect entropy in solutions.

\section{ACKNOWLEDGMENT}

Authors are thankful to Department of Chemistry for providing laboratory facilities. The authors are also thankful for the facilities and grants given under UGC-SAP for Department Research Support (DRS) and Department of Science and Technology (DST) New Delhi for Fund for Improvement of Science and Technology (FIST). 


\section{References}

[1] T. S. Straub. Tetrahedron Lett. 36 (1995) 663.

[2] S. Sandler, W. Karo, In Organic Functional Group Preparations 3 (1972) 372.

[3] S. N. López, et al., Bioorg. Med. Chem. 9 (2001) 1999.

[4] B. Baviskar, S. Patel, B. Baviskar, S. S. Khadabadi, M. Shiradkar, Asian J. Res. Chem. 1 (2008) 67.

[5] P. M. Sivakumar, S. Ganesan, P. Veluchamy, M. Doble, Chem. Biol. Drug. Des. 76 (2010) 407.

[6] F. Herencia, M. L. Ferrandiz, A. Ubeda, J. N. Domínguez, J. E. Charris, G. M. Lobo, M. J. Alcaraz, Bioorg. Med. Chem. Lett. 8 (1998) 1169.

[7] R. Kachadourian, B. J. Day, S. Pugazhenti, C. C. Franklin, E. Bastide, G. Mahaffey, C. Gauthier, A. Di-Pietro, A. Boumendjel, J. Med. Chem. 55 (2012) 1382.

[8] P. M. Sivakumar, T. D. Muthu Kumar, M .Toble, Chem. Biol. Drug Des. 74 (2009) 68.

[9] R. M. Mishra, A. Wahab, Ind. J. Hetero. Chem. 13 (2003) 29.

[10] T. Narsinghani, M. C. Sharma, S. Bhargav, Med Chem Res 22 (2013) 4059.

[11] C. A. Calliste, J. C. Le Bail, P. Trouillas, C. Pouget, G. Habrioux, A. J. Chulia, J. L. Duroux, Anticancer Res. 21(6A) (2001) 3949.

[12] T. Narender, T. Khaliq, S. Nishi, N. Goyal, S. Gupta, Bioorg. Med. Chem. 13 (2005) 6543.

[13] X. Wu, P. Wilairat, M. Go, Bioorg. Med. Chem. Lett. 12 (2002) 2299.

[14] A. Agarwal, K. Srivastava, S. K. Puri, M. S. Chauhan, Bioorg. Med. Chem. 13 (2005) 6226.

[15] P. M. Sivakumar, G. Babu, S. K. Mukesh, Chem. Pharm. Bull. 55 (2007) 44.

[16] Y. M. Lin, Y. Zhou, M. T. Flavin, L. M. Zhou, W. Nie, F.C. Chen, Bioorg. Med. Chem. 10 (2002) 2795.

[17] G. S, Viana, M. A Bandeira, F. J. Mantos, Phytomedicine 10 (2003) 189.

[18] Z. Nowakowska, Eur. J. Med. Chem. 42 (2007) 125.

[19] J. H. Cheng, C. F Hung, S. C. Yang, J. P. Wang, S. J. Won, C. N. Lin, Bioorg. Med. Chem. 16 (2008) 7270.

[20] S. Ducki, R. Forrest, J. A. Hadfield, A. Kendall, N. J. Lawrence, A.T. Mc-Gown, D. Rennison, Bioorg. Med. Chem. 8 (1998) 1051.

[21] G. Valdameri, et al., J. Med. Chem. 55 (2012) 3193.

[22] J. C. Jang, S. Lee, Y. Min, D. Lim, E. Jung, H. Oh, M. Oh, S. Jung, J. Med. Chem. 56 (2013) 6136.

[23] J. Gao, Z. W. Wang, D. M. Xu, R. K. Zhang, J. Chem. Eng. Data 52 (2007) 189.

[24] V. D. Athawale, P. Mathur, Experimental of Physical Chemistry 2001. 
[25] D. M. Aragon, M. A. Ruidiaz, E. F. Vargas, C. Bregni, D. A. Chiappetta, A. Sosnik, F. Martinez, J. Chem. Eng. Data 53 (2008) 2576.

[26] R. R. Krug, W. G. Hunter, R. A. Grieger, J. Phys. Chem. 80 (1976) 2341.

[27] P. Bustamante, S. Romero, A. Pena, B. Escalera, A. Reillo, J. Pharma. Sci. 87 (1998) 1590.

[28] P. S. Kalsi, Organic reactions and their mechanisms, New age international (P) limitedNew Delhi, 2nd edition, 2004, 119.

[29] A. A. El-Bindary, A. Z. El-Sonbati, E. H. El-Mosalamy, R. M. Ahmed, Chem. Pap. 57 (2003) 255.

[30] S. John Joseph, et al., International Letters of Chemistry, Physics and Astronomy 5 (2014) 99-123.

[31] Sekar K. G., Thirunarayanan G., International Letters of Chemistry, Physics and Astronomy 8(3) (2013) 249-258.

[32] Ranganathan K., et al., International Letters of Chemistry, Physics and Astronomy 4 (2012) 66-75.

[33] Arulkumaran R., et al., International Letters of Chemistry, Physics and Astronomy 5 (2013) 21-38.

[34] Sakthinathan S. P., et al., International Letters of Chemistry, Physics and Astronomy 6 (2013) 77-90.

[35] Sekar K. G., Thirunarayanan G., International Letters of Chemistry, Physics and Astronomy 8(2) (2013) 160-174.

[36] S. Vijayakumar, International Letters of Chemistry, Physics and Astronomy 9(1) (2013) 68-86.

[37] Thirunarayanan G., Sekar K. G., International Letters of Chemistry, Physics and Astronomy 10 (2013) 18-34.

[38] R. Sundararajan, et al., International Letters of Chemistry, Physics and Astronomy 1 (2014) 67-73.

[39] John Joseph S., et al., International Letters of Chemistry, Physics and Astronomy 4 (2014) 48-65. 